growth of a world society as the successor to the International Institute for Intellectual Co-operation and, more immediately, the Conference of Allied Ministers of Education.

"UNESCO and a World Society" shows how the Organisation is constituted, the function of its subdivisions and the range of its activities. All this is clearly set out, as is also the controversy between those who believed that the Organisation should be limited and concentrated for the intellectual few, and those who declared that to help the minority is not the way to save peace and that the Organisation is not for the pedants but for the people. The design of the Organisation shows how these differing opinions have been fairly reconciled.

Unesco has also been criticized for arranging meetings between men of science to discuss 'obscure' topics on the grounds that the sole purpose of the Organisation is to contribute to peace and security; the critics assert that the Organisation should undertake nothing that cannot be demonstrated on the blackboard to contribute directly to peace. Its position is defended in "the belief that everything contributing effectively to human sympathy through intellectual and spiritual forces is U.N.E.S.C.O.'s business". This being the case, one is tempted to ask why the Organisation has not concerned itself with those sports organisations which have already done so much to bring people of different countries together. Through the Anglo-Netherlands Sports Association, for example, contests of all kinds take place throughout the year between representatives of towns like Sheffield and Nijmegen, Hull and Rotterdam, and Rotherham and Amersfoort. During the interchanges much is done, both officially and unofficially, to give the guests a real impression of the educational, industrial, social, economjc and eivic life of the town they are visiting. Here is a popular educational movement of peoples which is directly contributing to understanding and which Unesco might develop for its own sake. More interest, too, might be shown in the rapidly developing schemes for the exchange of employees between industrial organisations like the members of the British Iron and Steel Federation and similar industries overseas.

The rest of the pamphlet contains details of the various committees, of work already accomplished by the Organisation and a summary of its immediate programme for Britain. It is a record of increasing activity which needs to be more widely known, and shows that the present publicity campaign will not have begun too early.

T. H. HAWKINS

\section{ANNUAL EXHIBITION OF THE ROYAL PHOTOGRAPHIC SOCIETY}

7 HE ninety-third Annual Exhibition of the Royal 1 Photographic Society is again being held in two parts. The second part, which contains the scientific exhibits, opened on October 9 at the Society's House, 16 Prince's Gate, S.W.7, and closed on October 30. The whole exhibition will be shown at the Museums and Art Gallery, New Walk, Leicester, during the period November 6-December 5 .

The section on Nature photography again contains many high-speed flash photographs, taken both by day and by night. The value of the method in studying fast movements is well shown by photographs of birds in flight and of the tongue action of toads and chameleons. It can also result in pictures displaying surprisingly beautiful designs. There is a photograph, of a robin alighting on a twig, in which the individual wing feathers are spread fanwise in a manner that could not be seen in any other way. (This photograph, with many others from the Exhibition, is reproduced in "The Year's Photography", the September issue of the Photographic Journal.) of special interest are the several instructive series showing the life-histories of various creatures and plants. These are all well annotated; but the descrip. tions of the life and habits of the Natal Peripatus and of the one-day life of the stinkhorn deserve special mention. It is remarkable how much the presence of a caption, even on an individual picture, increases the interest. There is a picture of a young grey seal among many other equally superb pictures which remains in the writer's memory simply because it is the one with a short descriptive caption.

Many of the scientific photographs are liberally provided with descriptive matter, but there are woeful exceptions. Among these are a pair of excellent photomicrographs of the living spermatozoa of a mouse, one taken with phase contrast and the other by ultra-violet light. Others in the same series show the chromosomes in bean-root cells at different stages in mitosis. These are presumably phase-contrast pictures; but it is not clear in the catalogue, and only the catalogue numbers appear on the exhibits. Another pair of pictures illustrates normal and pathological nerve cells (according to the catalogue), but there is no indication of which is which. Information is also entirely lacking in several pictures labelled "Molecular Design". The writer presumes that these were intended for the pictorial part of the Exhibition-they have no scientific implication as shown.

There is an excellent series comparing results obtained with different kinds of microscope-normal optical, phase-contrast, interference and electron. Another exhibit describes a method of recording the colour aberrations of high-power objectives. A straight-line obj $\Leftrightarrow$ ct illuminated by mercury-cadmium light produces an elongated line spectrum on the photographic plate by means of a grating and prism placed behind the eyepiece. The line object is tilted at a small angle to the normal object plane, so that the position of sharp focus of the spectrum lines of different wave-length varies according to the chromatic aberrations of the system.

Two exhibits illustrate a new method of microradiography applied to the investigation of complex alloyed steels. Variations in composition give rise to a fine banded structure, and the identification of the elements responsible is possible by selection of the exposing radiation. Another series, illustrating a technique for resolution testing in autoradiography, demonstrates the possibilities of the autoradingraphy of plant or animal tissue containing radioactive tracers. Pinhole radiographs of the internal surface of the copper anode of a magnetron enables the distribution, direction and speed of the bombirding electrons, about which little is known in detail, to be determined (see Nature, 161, 244 ; Feb. 14, 1948).

Nuclear plate photographs illustrate the natural disintegration of radium and radiothorium, a meson track $400 \mu$ long and the effects of selective development on nuclear tracks. Lowering the $p H$ of the developer reduces the background relative to the tracks, and addition of bromide restrains the development of alpha tracks before affecting fission tracks. 
Among the exhibits deserving special mention is one of lightning flashes taken during a thunderstorm. Owing to the Clayden effect, a flash occurring at the beginning of the exposure appears as a black line on a grey background, while one occurring at the end of the exposure is white. Some high-speed oscillograms demonstrate that it is now possible to record photographically traces made at a writing speed no less than four per cent of the speed of light. There are also some fine cloud studies and a number of large-scale photographs of geological formations taken from the air.

Some of the exhibits are also reproduced as lantern slides, which provide an interesting comparison with the prints. Unfortunately, they are on different floors. The lantern slides also include some beautiful colour transparencies of crystals in polarized light and of photo-elastic stress patterns of loaded beams and other structures.

The medical photographs are not numerous, which is perhaps just as well for the visitor's peace of mind in view of the extreme degree of clarity achieved in the reproduction of detail.

\section{PHYSICAL CHEMISTRY IN PHARMACY}

$A^{1}$

T the British Pharmaceutical Conference held at Brighton in August, the chairman, Dr. N. Evers, gave an address entitled "The Importance of Physical Chemistry in Pharmacy". He claimed that the importance of this aspect of pharmacy has not been sufficiently appreciated in the past and that the pharmacist requires a wide knowledge of physical and chemical properties of materials. Dr. Evers said the pharmaceutical industry is probably concerned with a wider range of materials than any other, even including the food industry; and that the most important asset which the pharmacist possesses as a result of his training and experience is his familiarity with the properties of materials and his knowledge of their behaviour over a range of different circumstances.

In recent years an ever-widening range of new materials has been offered for the use of the phar. macist by the synthetic chemist; substances which, if used with knowledge and skill, can be made to produce almost any desired properties in the product. In order to make the most effective use of these substances, the pharmacist requires a knowledige of the relevant physical chemistry. It should not be necessary to rely on the manufacturer of such products for suggesting suitable formulæ, because only the user is in a position to know exactly what he requires of his product. It is all the more desirable, therefore, that he should possess the knowledge of physical chemistry which will enable him to produce new preparations by the use of such substances, either for the presentation of new drugs or for new methods of presenting old ones.

\section{Applications of Physical Chemistry}

Without introducing examples which are recondite or unusual, any attempt to make a list of physicochemical phenomena which are encountered by the pharmacist could not fail to include the following items :

\author{
Solubility \\ Iouization \\ Hydrogen ion \\ Mass action and reaction velocity \\ Osmotic pressure \\ Osmotic
Dirtysis \\ Surface tension \\ Emulsification \\ Vapour pressure \\ Dififusion \\ Colloids \\ Adsorption
}

This list does not include the applications of physical chemistry in analytical chemistry, but only phenomena which may be encountered in pharmaceutics.

Large-scale manufacturing pharmacy employs many physico-chemical processes such as evaporation, distillation and drying. The design of plant for such processes involves problems which can safely be left to the chemical engineer; but the pharmacist who uses the products must have a sufficient knowledge of the underlying principles of the processes used to enable him to make a choice of the most suitable method from the point of view of both the effect on the product and of cost. For example, in a new process of drying, such as freeze-drying, there may be no previous experience to guide him, and each problem requires its own solution to be worked out from physico-chemical considerations.

The use of adsorbents in the pharmaceutical industry seems to provide endless problems for research. The purification of active principles by adsorption is not a process which has been much developed. It was used in the carbon adsorption process for penicillin, where incidentally it was found that comparatively few types of carbon were really effective adsorbents. Carbon has been used for the adsorption of pyrogens and 'Permutit' for the removal of traces of adrenalin from cortical extracts. Pussibly the new base-exchange resins may have some uses in this type of work. They have been used, for example, for the conversion of calcium penicillin to sodium penicillin. Chromatographic separation is not particularly suitable for large-scale work, and probably has its chief uses in the research laboratory and in the purification of highly active substances which are only produced in small quantities.

The sterile filtration of injections bristles with physico-chemical problems mainly connected with the adsorption of active principles by the filtering medium. Even sintered glass is not entirely guiltless in this respect, and there are instances where no method of sterile filtration can be used without loss of potency. There seems no doubt that in some cases the filtering medium exerts a chromatographic effect, and that an initial adsorption may occur which is afterwards washed out.

\section{Rheology}

The science of rheology is defined by its chief exponent, Dr. G. W. Scott Blair, as concerned with the "deformation and flow of matter". The two lectures given by Dr. Scott Blair recently to the Pharmaceutical Society gave an indication of the scope of rheology and its importance in pharmacy. It is concerned with the properties of solids and liquids such as viscosity, surface tension, thixotropy, elasticity, hardness or softness, and those vague attributes which we know as 'consistency', 'firmness', 'springiness', 'toughness', 'tackiness', 'body' and other such terms. Many of these properties are not measurable in a quantitative manner, and the human 